\title{
Cerebral Edema in Diabetic Ketoacidosis - Fluid Shifts and Shifting Paradigms
}

\author{
Adrian Araya ANd Ram K Menon* \\ Pediatric Endocrinology, CS Mott Children's Hospital, University of Michigan, \\ Ann Arbor, Michigan, USA. *rammenon@med.umich.edu
}

$\mathrm{D}$ iabetic ketoacidosis (DKA) is the most significant acute complication of type 1 diabetes mellitus (T1DM). DKA is a common presentation for new onset T1DM, occurring in up to $25 \%-40 \%$ of newly diagnosed children [1-3]. The essential components of the treatment regimen for DKA are fluid and electrolyte resuscitation and insulin therapy. Cerebral edema manifests clinically in approximately $1 \%$ of DKA patients with progression to brain herniation, representing the major cause of mortality and morbidity in DKA [4]. However, asymptomatic or subclinical cerebral edema, as detected by subtle brain computed tomography (CT) or magnetic resonance imaging (MRI) changes, can occur in as much of $50 \%$ of DKA patients. Hence, early identification of clinically relevant cerebral edema in DKA relies on clinical criteria rather than solely on radiological tests [5]. Clinical identification of cerebral edema is further confounded by alterations in mental status that are part of the clinical picture of DKA $[4,5]$. In DKA, cerebral edema usually occurs early (occurring within the first 7-8 hours in approximately $2 / 3 \mathrm{rd}$ ) in the treatment of DKA with the remaining cases occurring up to 28-30 hours after fluid resuscitation and initiation of insulin treatment $[3,6]$. Notably, cerebral edema has also been reported, albeit rarely, prior to initiation of fluid resuscitation and treatment.

The mechanism(s) of cerebral edema in DKA continue to be debated and explored. The traditional model ascribes cerebral edema to retention of cerebral intracellular osmolytes causing fluid shifts into the intracellular space. Acceptance of this model resulted in an emphasis on rate and composition of intravenous fluid administration as significant parameters to mitigate the risks of cerebral edema. This dogma is being challenged by newer studies indicating role for vasogenic edema from blood-barrier destruction, and cytotoxic edema from ischemia in the development of cerebral edema in DKA. Furthermore, a recent landmark randomized control study [6] revealed no differences between intravenous administration of $0.45 \%$ vs $0.9 \%$ normal saline at either rapid or slow infusion rates in children with DKA with Glasgow coma scale (GCS) $>11$. One caveat of this study is the exclusion of sicker children with GCS $<11$, due to obvious ethical concerns [6]. Risk factors predisposing to cerebral edema include new onset T1DM, younger age ( $<5$ years) and markers of severe DKA such as higher serum urea nitrogen concentrations, severe acidemia $(\mathrm{pH}<7.1)$, lower initial bicarbonate and lower partial pressures of arterial $\mathrm{CO}_{2}(<20 \mathrm{~mm} \mathrm{Hg})[2,4]$.

The study by Agarwal, et al. [7] in this issue of Indian Pediatrics highlights many of the factors that are known to be associated with increased risk for cerebral edema, such as lower partial pressures of arterial $\mathrm{CO}_{2}$ and new onset diabetes. However, an unusual finding in this study [7] is the extraordinarily high rate $(24.3 \%)$ of clinically diagnosed cerebral edema. Contemporary literature describes clinically apparent cerebral edema in $\sim 1 \%$ of episodes of DKA [1,5]. In the current study, the diagnostic criterion used in $15(68 \%)$ patients for diagnosing cerebral edema was abnormal central breathing pattern. It is not clear whether this criterion was applied at the time of diagnosis of DKA or after initiation of treatment. In the absence of the other diagnostic criteria (abnormal motor or verbal response to pain, decorticate or decerebrate posture, or cranial nerve palsy, especially involving III, IV, and VI cranial nerves) [5], Kussmaul breathing, a classic sign of DKA at presentation, would be difficult to differentiate from the cerebral edema criterion of abnormal central breathing. It is noteworthy that the rate of cerebral edema development during treatment was much lower at $4.7 \%$. This study also highlights the importance of having complete information about fluid resuscitation in the transfer of care documents, since history of prior fluid treatment was a predictive factor for cerebral edema, especially in the cases where it developed after initiation of treatment post-admission. The development of cerebral edema in one of their patients 60 hours after initiation of treatment is unusual and exemplifies the need for continuous assessment by trained practitioners in 
identifying changes in neurological status in these patients.

The only way to completely prevent cerebral edema in DKA is to avoid DKA. The study by Agarwal, et al. [7] highlights the critical importance of early diagnosis of new onset T1DM before the patient has progressed to DKA. In countries such as India, with a diverse heathcare delivery system, primary healthcare providers including community health care workers should be constantly vigilant to the possibility of the diagnosis of new-onset T1DM in a child presenting with suggestive symptoms such as polyuria (especially new onset nocturia or bed wetting), polydipsia, and weight loss.

Funding: None; Competing interest: None stated.

\section{REFERENCES}

1. Bialo SR, Agrawal S, Boney CM, Quintos JB. Rare complications of pediatric diabetic ketoacidosis. World $\mathrm{J}$ Diabetes. 2015;6:167-74.
2. Glaser N, Barnett P, McCaslin I, et al. Risk factors for cerebral edema in children with diabetic ketoacidosis. $\mathrm{N}$ Engl J Med. 2001;344:264-9.

3. Wolfsdorf J, Glaser N, Sperling MA. Diabetic Ketoacidosis in Infants, Children, and Adolescents. A Consensus Statement from the American Diabetes Association. Diabetes Care. 2006;29:1150-9.

4. Long B, Koyfman A. Emergency medicine myths: Cerebral edema in pediatric diabetic ketoacidosis and intravenous fluids. J Emerg Med. 2017;53:212-21.

5. Muir AB, Quisling RG, Yang MC, Rosenbloom AL. Cerebral edema in childhood diabetic ketoacidosis: Natural history, radiographic findings, and early identification. Diabetes Care. 2004;27:1541-6.

6. Kuppermann N, Ghetti S, Schunk JE, Stoner MJ, Rewers A, McManemy JK, et al. Clinical trial of fluid infusion rates for pediatric diabetic ketoacidosis. $\mathrm{N}$ Engl J Med. 2018;378:2275-87.

7. Agarwal N, Dave C, Patel R, Shukla R, Kapoor R, Bajpai A. Factors associated with cerebral edema at admission in Indian children with diabetic ketoacidosis. Indian Pediatr. 2020;57:310-3. 